\title{
Trajectory Planning for 6-DOF Robotic Arm Based on Quintic Polynormial
}

\author{
Xiaojie Zhao ${ }^{1,2}$, Maoli Wang, ${ }^{1,2}$, Ning Liu $^{3}$ and Yongwei Tang, ${ }^{1,2}$ \\ ${ }^{1}$ Shandong Computer Science Center (National Supercomputer Center in Jinan), Jinan 250014, China \\ ${ }^{2}$ Shandong Provincial Key Laboratory of Computer Networks, Jinan 250014, China \\ ${ }^{3}$ School of automation engineering, Qufu Normal University, Rizhao, 276826, China \\ *Corresponding author
}

\begin{abstract}
The paper deals with research on polynomial trajectory planning for 6-DOF robotic arm. On the basis of kinematics analysis, we carried out trajectory planning and simulation of joint space. Through the trajectory planning diagrams of MATLAB simulation on the two methods, the results showed that quintic polynomial is better than cubic polynomial in the effect of planning. As a result, this method used in six degrees of freedom industrial robot trajectory planning is feasible.
\end{abstract}

Keyword-robotic arm; trajectory planning; 6-DOF; polynomial

\section{INTRODUCTION}

With the development of the economy, the application of manipulator is more and more extensive, and its technical requirements are getting higher and higher. Using the mechanical arm operation can improve the quality and quantity of the products, increase productivity and reduce cost while trajectory planning directly affects the quality of the robot's work $^{[1]}$. The trajectory refers to the displacement, velocity and acceleration of the manipulator in motion. Calculating the expected trajectory is trajectory planning which is based on the requirements of the task ${ }^{[2]}$. The requirement of the trajectory planning is to control the movement speed of the manipulator during the movement and the movement space is always kept within the allowable range of each joint movement. The running trajectory is smooth and continuous [3]. The mechanical arm operation experiment is carried out by using MATLAB Robotics Toolbox ${ }^{[4]}$. It turns out that the toolbox can simulate a robot. If you choose different types of joint interpolation functions under the required constraints, you can generate different trajectories in the joint space for trajectory planning.

Using cubic polynomials and seven polynomials to trajectory planning has some drawbacks. In order to meet the requirement that 6-DOF robotic arm should run a smooth, continuous trajectory during the operation, we will use quintic polynomial. MATLAB simulation verifies the feasibility of the method.

\section{KinEMATICS ANALYSIS}

The coordinate frame of link of 6-DOF series robotic arm is shown in Figure 1. The D-H parameters are shown in Table I.

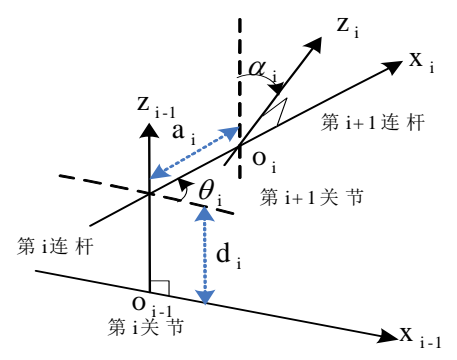

FIGURE I. THE COORDINATE FRAME OF LINK

TABLE I. THE D-H PARAMETERS

\begin{tabular}{|c|c|c|c|}
\hline$\left.\theta_{\mathrm{i}} / \mathbf{(}^{\circ}\right)$ & $\alpha_{\mathrm{i}-1} /\left(^{\circ}\right)$ & $\mathrm{a}_{\mathrm{i}-1} /(\mathbf{m m})$ & $\mathrm{d}_{\mathrm{i}} /(\mathbf{m m})$ \\
\hline$\theta_{1}$ & $0^{\circ}$ & 0 & 0 \\
\hline$\theta_{2}$ & $-90^{\circ}$ & 0 & 280 \\
\hline$\theta_{3}$ & $0^{\circ}$ & 800 & 0 \\
\hline$\theta_{4}$ & $-90^{\circ}$ & 60 & 700 \\
\hline$\theta_{5}$ & $90^{\circ}$ & 0 & 0 \\
\hline$\theta_{6}$ & $-90^{\circ}$ & 0 & 120 \\
\hline
\end{tabular}

\section{A. Positive Kinematics Analysis}

The so-called positive kinematics is that the angles and geometric parameters of the mechanical arm are known whereby the position of the robot end actuator relative to the reference coordinate system. Positive kinematics is an indispensable step in the display of the movement trajectory of the mechanical arm. A homogeneous transformation matrix i-1Ti can be determined between the adjacent link i and the link (i-1) for the 6-DOF series robotic arm.

$$
{ }^{i-1} T_{i}=\left[\begin{array}{cccc}
c \theta_{i} & -s \theta_{i} & 0 & a_{i-1} \\
s \theta_{i} c \alpha_{i-1} & c \theta_{i} c \alpha_{i-1} & -s \alpha_{i-1} & -d_{i} s \alpha_{i-1} \\
s \theta_{i} s \alpha_{i-1} & c \theta_{i} s \alpha_{i-1} & c \alpha_{i-1} & d_{i} c \alpha_{i-1} \\
0 & 0 & 0 & 1
\end{array}\right]
$$

The transformation matrix $\mathrm{T} 6$ of the mechanical arm coordinate system $\{6\}$ with respect to the base $\{0\}$ can be obtained by sequentially multiplying the homogeneous transformation matrix $\mathrm{i}-1 \mathrm{Ti}$ in sequence. We can get a matrix based on $\theta_{i}$ according to Table 1 and the formula (1). 


\section{B. Inverse Kinematics Analysis}

The inverse kinematic analysis of robots is the basis of the planning and control of the mechanical arm movement. The value of each joint angle $\theta_{i}$ is obtained by the known end-effector, which is relative to the position and linkage parameters of the reference coordinate system.

Take $\theta_{1}$ as an example, Multiplied by the inverse of ${ }^{0} T_{1}$ on left side of the equation ${ }^{0} T_{6}={ }^{0} T_{1}^{1} T_{2}^{2} T_{3}^{3} T_{4}^{4} T_{5}^{5} T_{6}$ which is ${ }^{0} T_{1}{ }^{10} T_{6}={ }^{1} T_{2}^{2} T_{3}^{3} T_{4}^{4} T_{5}^{5} T_{6}$, the inverse of ${ }^{0} T_{1}$ is equal to its transpose.

$$
{ }^{0} T_{1}^{-1}={ }^{0} T_{1}^{\prime}=\left[\begin{array}{cccc}
c_{1} & s_{1} & 0 & 0 \\
0 & 0 & 1 & 0 \\
s_{1} & c_{1} & 0 & 0 \\
0 & 0 & 0 & 1
\end{array}\right]
$$

Then we derive

$$
\left[\begin{array}{cccc}
c_{1} & s_{1} & 0 & 0 \\
0 & 0 & 1 & 0 \\
s_{1} & c_{1} & 0 & 0 \\
0 & 0 & 0 & 1
\end{array}\right] \times\left[\begin{array}{cccc}
n_{x} & o_{x} & a_{x} & p_{x} \\
n_{y} & o_{y} & a_{y} & p_{y} \\
n_{z} & o_{z} & a_{z} & p_{z} \\
0 & 0 & 0 & 1
\end{array}\right]=T_{2}{ }^{2} T_{3}{ }^{3} T_{4}{ }^{4} T_{5}{ }^{5} T_{6}
$$

Expanding the formula and according to the principle of equality on both sides of the matrix, we derive

$$
-s_{1} p_{x}+c_{1} p_{y}=d_{2}
$$

Using triangular substitutions:

$$
p_{x}=\rho \cos \phi, p_{y}=\rho \sin \phi
$$

When $\rho=\sqrt{p_{x}^{2}+p_{y}^{2}}, \varphi=a \tan 2\left(p_{x}, p_{y}\right)$. Substituting (5) into (4), we can get the solution of $\theta_{1}$ :

$$
\theta_{1}=a \tan 2\left(p_{y}, p_{x}\right)-a \tan 2\left(d_{2}, \pm \sqrt{p_{x}^{2}+p_{y}^{2}-d_{2}^{2}}\right)
$$

The positive and negative signs correspond to the two possible solutions.

Similarly, we can get the value of other $\mathrm{x}$. Usually following the principle of "move the small joints more, move the large joints less" to select the most satisfying set of solutions to meet the requirements of the robotic arm.

\section{Joint Space Trajectory PlanNing}

The task of the robotic arm trajectory planning is to design the motion function of each joint of the arm based on certain tasks to be accomplished by its end actuators. We can select different joint interpolation functions to generate different trajectories.

\section{A. Cubic Polynomial Interpolation}

We define the start point of the robotic arm joint movement is $t_{0}$, the start point of the joint angle is $\theta_{0}$, the end point is $t_{\mathrm{f}}$, the joint angle is $\theta_{f}$.The description of the trajectory can be represented by a smooth interpolation function of the start angle and end angle. Assume that the joint speed at both times is known and set to zero. Then, in order to achieve a smooth motion of a single joint, the trajectory function $\theta(t)$ satisfies at least four constraints:

$$
\left\{\begin{array}{c}
\theta(0)=\theta_{0} \\
\theta\left(t_{\mathrm{f}}\right)=\theta_{f} \\
\dot{\theta}(0)=0 \\
\dot{\theta}\left(t_{\mathrm{f}}\right)=0
\end{array}\right.
$$

The above constraint determines the functional equation of the joint angle and velocity:

$$
\left\{\begin{array}{c}
\theta(t)=a_{0}+a_{1} t+a_{2} t^{2}+a_{3} t^{3} \\
\dot{\theta}(t)=a_{1}+2 a_{2} t+3 a_{3} t^{2}
\end{array}\right.
$$

We bring the constraint (7) into the equation (8). Solving the equation we can derive

$$
\left\{\begin{array}{c}
a_{0}=\theta_{0} \\
a_{1}=0 \\
a_{2}=\frac{3}{t_{f}^{2}}\left(\theta_{f}-\theta_{0}\right) \\
a_{3}=-\frac{2}{t_{f}^{3}}\left(\theta_{f}-\theta_{0}\right)
\end{array}\right.
$$

\section{B. Quintic Polynomial Interpolation}

In the case where the trajectory is more stringent and the constraint condition is increased, the cubic polynomial interpolation can't satisfy the requirement, and the high order polynomial is used for interpolation. For example, when the starting point and the ending point of a certain path are specified for the position, velocity and acceleration of their joints, a quintic polynomial can be used for interpolation. The functional equation of the joint angle, velocity and acceleration is as follows:

$$
\left\{\begin{array}{c}
\theta(t)=a_{0}+a_{1} t+a_{2} t^{2}+a_{3} t^{3}+a_{4} t^{4}+a_{5} t^{5} \\
\dot{\theta}(t)=a_{1}+2 a_{2} t+3 a_{3} t^{2}+4 a_{4} t^{3}+5 a_{5} t^{4} \\
\ddot{\theta}(t)=2 a_{2}+6 a_{3} t+12 a_{4} t^{2}+20 a_{5} t^{3}
\end{array}\right.
$$


Assuming six conditions are known, i.e., $\theta\left(t_{0}\right)=\theta_{0}: \theta\left(t_{f}\right)=\theta_{f}$ $\dot{\theta}\left(t_{0}\right)=\ddot{\theta}\left(t_{0}\right)=\dot{\theta}\left(t_{f}\right)=\ddot{\theta}\left(t_{f}\right)=0$.We bring these known conditions into the form (10) and get the coefficients :

$$
\left\{\begin{array}{c}
a_{0}=\theta_{0} \\
a_{1}=0 \\
a_{2}=0 \\
a_{3}=\frac{10\left(\theta_{f}-\theta_{0}\right)}{t_{f}^{3}} \\
a_{4}=\frac{-15\left(\theta_{f}-\theta_{0}\right)}{t_{f}^{4}} \\
a_{5}=\frac{6\left(\theta_{f}-\theta_{0}\right)}{t_{f}^{5}}
\end{array}\right.
$$

\section{Simulation Results}

Set starting point $\mathrm{p} 0=[970,0,1140]$ and the target point $\mathrm{p} 1=$ [611.069, 386.390, -19.873], in the working space of the known robotic arm. The joint angle values of the joints at two points are obtained by the ikine function in MATLAB Robotics Toolbox as shown in Table II. Using the link function to construct the FS20N 3D model, its position and orientation at different points are shown in Figure 2.

\section{TABLE II. THE JOINT ANGLE VALUES OF THE JOINTS AT TWO} POINTS

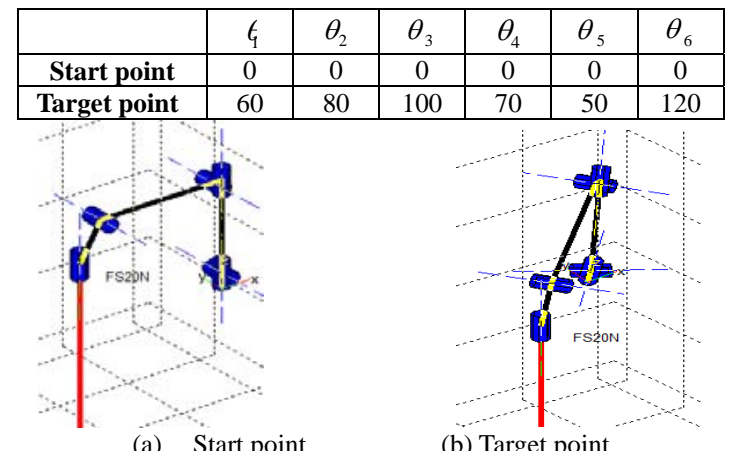

FIGURE II. THE POSITION OF THE JOINTS AT TWO POINTS

The motion planning of the manipulator from the start point to the target point is realized by the cubic polynomial interpolation and the quintic polynomial polynomial interpolation method respectively. The angle, velocity, acceleration curve is also obtained. The start point and target point of both approaches are zero, and the acceleration of the start point and target points is zero too.

Figure 3 shows the angular displacement, angular velocity and angular acceleration curve of each joint obtained by cubic polynomials(The joints in the figure from top to bottom are: joint 6 , joint 3 , joint 2 , joint 4 , joint 1 , joint 5 ).It can be seen from the figure that the angular displacement function, angular velocity function and angular acceleration function of each joint are continuous, the movement of the robot in this movement is smooth. In Fig. 4, the joint curves obtained by the quintic polynomial are smoother and smoother than the joint curves obtained by cubic polynomials, the joint acceleration curves are not straight but curved, and have better effect.

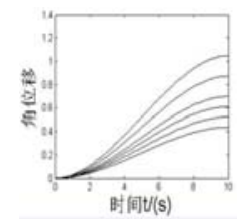

(a) displacement

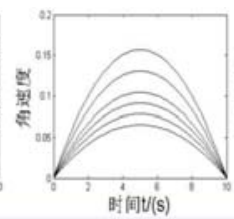

(b) velocity

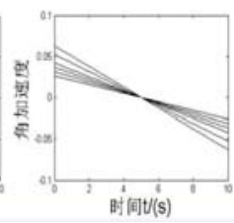

(c) acceleration
TABLE III. CUBIC POLYNOMIAL JOINT CURVES

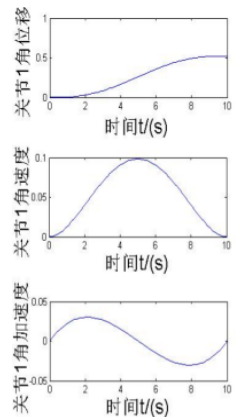

(a) joint 1
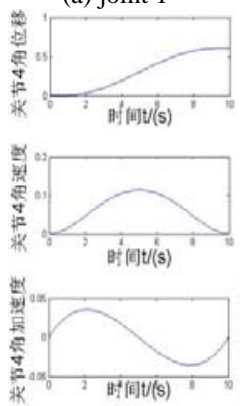

(d) joint 4

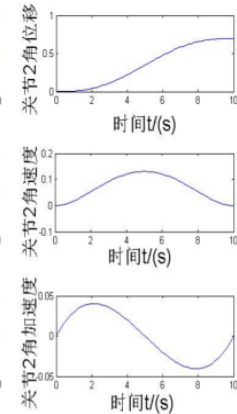

(b) joint 2
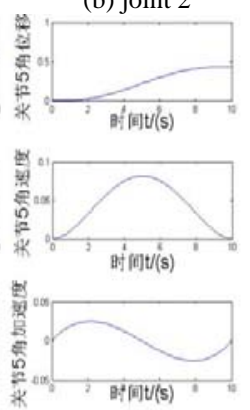

(e) joint 5
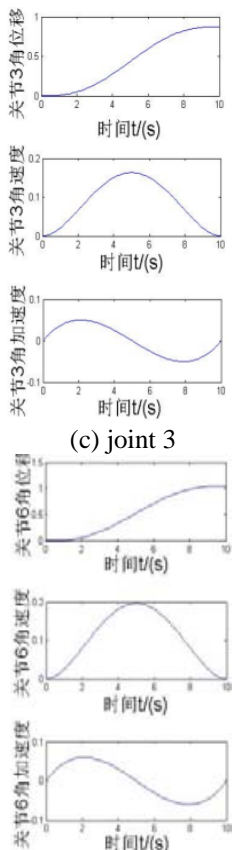

(f) joint 6 (c) joint 3

TABLE IV. QUINTIC POLYNOMIAL $1 \sim 6$ JOINT TRAJECTORY CURVE

\section{CONCLUSIONS}

In this paper, we carry out the kinematics analysis of 6-DOF robotic arm, and the application of cubic polynomial and quintic polynomials in 6-DOF robotic arm trajectory planning is emphasized. Finally, we obtained the corresponding curve by MATLAB simulation. After comparing and analyzing, the results show that the quintic polynomials method has more obvious effect on the trajectory planning of the 6-DOF robotic arm.

We obtained smooth and continuous trajectory planning curve through quintic polynomials, indicating that this method is feasible in the application of trajectory planning of similar 6-DOF robotic arm. This method can make the robotic arm to run smoothly, reduce the occurrence of jitter and vibration, improve the stability of system, prolong the life of robotic arm. It can provides a reference for improving the smooth trajectory planning of robotic arm.

\section{ACKNOWLEDGEMENT}

This research was financially supported by National Science and Technology Major Project (2014ZX04015011); 
Shandong Province Major Special Support Funds (2015ZDZX10002); Shandong Province Major Special Support Funds (2015ZDXX0101G05).

\section{REFERENCES}

[1] YuYang Fu,6R.Research on Structural Design and Motion Trajectory Planning of Industrial Robot [D].Master Thesis of Wuyi University.2013.

[2] ZiXing Cai, Robotics [M]. Beijing: Tsinghua University Press, 2009: 264.

[3] LinShi Gao,XiaoLin Liu And YuanXian Ou,Robotic arm smoothing programming algorithm based on quintic polynomials[J],Manufacturing Automation, 2013, (11): 16-17, 24.

[4] Saeed B.Niku. Introduction to Robotics [M]. BEIJING: Publishing House of Electronics Industry, 2013.

[5] Bin Xie, ZiXing Cai,Simulation experiment teaching of Robotics Based on MATLAB Robotics Toolbox [J],Computer Education,2010 